\title{
Fractal Dimension as Quantifier of EEG Activity in Driving Simulation
}

\author{
$\mathrm{M}^{\mathrm{a}}$ Victoria Sebastián ${ }^{1, *\left(\mathbb{D}, \mathrm{M}^{\mathrm{a}} \text { Antonia Navascués }\right.}{ }^{2}$, Antonio Otal ${ }^{1}$, Carlos Ruiz $^{1}, \mathrm{M}^{\mathrm{a}}$ Ángeles Idiazábal $^{3}$, \\ Leandro L. Di Stasi ${ }^{4}$ and Carolina Díaz-Piedra ${ }^{4}$ (D)
}

1 Centro Universitario de la Defensa de Zaragoza, Academia General Militar, Ctra. Huesca s/n, 50090 Zaragoza, Spain; aotal@unizar.es (A.O.); cruizl@unizar.es (C.R.)

2 Department. Matemática Aplicada, Escuela de Ingeniería y Arquitectura, Universidad de Zaragoza, C/María de Luna 3, 50018 Zaragoza, Spain; manavas@unizar.es

3 Instituto Neurocognitivo Incia, Centro Adscrito a la Universidad de Barcelona, C/Balmes 203, 08006 Barcelona, Spain; instituto.incia@gmail.com

4 Instituto Mind, Brain, and Behavior Research Center-CIMCYC, University of Granada, Campus de Cartuja s/n, 18071 Granada, Spain; distasi@ugr.es (L.L.D.S.); dipie@ugr.es (C.D.-P.)

* Correspondence: msebasti@unizar.es; Tel.: +34-976739851

Citation: Sebastián, M.V.; Navascués, M.A.; Otal, A.; Ruiz, C.; Idiazábal, M.Á.; Di Stasi, L.L.; Díaz-Piedra, C. Fractal Dimension as Quantifier of EEG Activity in Driving Simulation. Mathematics 2021, 9, 11. https:// doi.org/10.3390/math9111311

Academic Editor: Duarte Pedro Mata de Oliveira Valério

Received: 24 March 2021

Accepted: 3 June 2021

Published: 7 June 2021

Publisher's Note: MDPI stays neutral with regard to jurisdictional claims in published maps and institutional affiliations.

Copyright: (c) 2021 by the authors. Licensee MDPI, Basel, Switzerland. This article is an open access article distributed under the terms and conditions of the Creative Commons Attribution (CC BY) license (https:// creativecommons.org/licenses/by/ $4.0 /)$.

\begin{abstract}
Dynamical systems and fractal theory methodologies have been proved useful for the modeling and analysis of experimental datasets and, in particular, for electroencephalographic signals. The computation of the fractal dimension of approximation curves in the plane enables the assignment of numerical values to bioelectric recordings in order to discriminate between different states of the observed system. The procedure does not require the stationarity of the signals nor extremely long segments of data. In previous works, we checked that this parameter is a good index for brain activity. In this paper, we consider this measurement in order to quantify the geometric complexity of the brain waves in states of rest and during vehicle driving simulation in different scenarios. This work presents evidence that the fractal dimension allows the detection of the brain bioelectric changes produced in the areas that carry out the different driving simulation tasks, increasing with their complexity.
\end{abstract}

Keywords: fractal dimension; electroencephalogram; driving simulation; interpolation

\section{Introduction}

Dynamical systems methodologies, and specifically those of fractal sets, have been shown to be useful for modeling and quantifying experimental data, particularly for neurological signals [1,2]. Information on brain activity can be obtained from oscillations in the electrical potential of the human brain. That means that the activity produced by brain neurons is extracted by means of electrodes located on the scalp or at the base of the skull. The first recording of this electrical activity in humans, known as an electroencephalogram (EEG), was carried out in 1924 by the German neurologist Hans Berger, who is considered as the father of electroencephalography. EEG recordings are non-stationary signals [3], so a precise analysis of them requires processing techniques that allow reflecting the intrinsic characteristics of these signals, as well as the time variations of their properties.

These signals, with their different frequencies, amplitudes, and shapes, may have a physiological or pathological value, but the relationships between abnormal EEG and brain functions and disorders are not yet well understood. Therefore, it is necessary to obtain quantitative methods that assign numerical values to the basic characteristics of the signal in order to define quality descriptors that determine the characteristics of the EEG and allow the discrimination between different physiological states and establish comparisons between several records [4].

The primarily techniques used in the analysis of EEG signals were the Fast Fourier Transform (FFT) or the Reduced Time Fourier Transform (STFT) [5]. These methods of time- 
frequency representation revealed the temporal evolution of the spectral characteristics of the signal.

Advances in nonlinear dynamics have made it possible to consider brain electrical activity modeling as a dynamical system and, in particular, as a chaotic-type dynamical system. The EEG signal is the gate to measure the complexity of the nonlinear behavior of brain bioelectric activity. Among the quantifiers of the complexity of the data of the EEG signals proposed are the Lyapunov exponents (LE), the largest Lyapunov exponent (LLE) or the correlation dimension, which is also called D2. These methods have the disadvantage that their calculation requires working with long data samples, and the computational time used is excessive. In addition, both D2 and LLE have been considered poor indices to discriminate between different mental tasks [6].

Our team has developed advanced computational and analytical methods with low computational cost to compute different quantizers of EEG signals. In particular, we use the fractal dimension to measure the complexity of the signal [7]. In general, the greater the dimension, the greater the complexity. This parameter makes it possible to investigate the brain electrogenesis of the signals and extract information that would not be discovered by mere visual inspection. The fractal dimension is a parameter that does not require any hypothesis of signal stationarity and does not need too long records and, therefore, allows the assignment of numerical values to the EEG records to discriminate between different brain states. Previous studies have applied this quantifier to analyze EEG signals in different situations [8-11]. Finotello et al. [12] extracted the EEG characteristics in sleep records using the fractal dimension. In their study, Ruiz and Molina [13] used the fractal dimension to study the relationship between cortical and cardiac dynamics and their role in the perception of emotions.

Our research group has used this quantifier to analyze attention/concentration during the performance of tasks with different difficulty [14-16]. Attention, one of the last complex brain processes to acquire the category of higher brain function, is the foundation of many other complex cognitive processes. It can be defined as selective and directed perception, interest in a particular source of stimulation and effort, or concentration on a task. The level of performance in a task and the ability to process information are dependent on the state of brain activation in which the subject is [17]. This activation can be studied by recording the oscillations of the electrical potential of the brain using the electroencephalogram, which allows to analyze the overload, attention, and concentration required during the performance of different tasks, as well as their loss.

In this work, the fractal dimension has been used to quantify the geometric complexity of brain waves in a group of subjects belonging to the Spain Armed Forces in states of rest and during the simulation of driving a military vehicle task in scenarios with different levels of difficulty.

It is hypothesized whether this fractal parameter can discriminate between the basal states with eyes open and closed and the simulated driving tests, showing the increase in cortical activity when moving from the resting states to the driving tasks. In addition, it is intended to indicate the areas of greatest activity in each of the driving tasks.

\section{Materials and Methods}

\subsection{Methods}

The fractal dimension of a curve is a dimensionless parameter that indicates the density of a set in the metric space where it is located; that is, its dimension as a geometric object. In the case of electroencephalographic signals, this dimension provides a measure of the complexity of each of the recording channels, allowing comparisons between them, as well as between groups and types of EEG.

The calculation of this quantifier can be simplified, since it is not an excessively restrictive parameter in terms of signal stationarity hypotheses, and it does not need too long records. Our team has developed several low-cost computational algorithms for calculating the fractal dimension, for example, from the reconstruction of the EEG signal 
using fractal interpolation functions built with systems of iterated functions [18,19]. In this work, a procedure has been implemented to calculate the fractal dimension $D$ of the trace of each EEG channel as a curve of the real plane, using the existing relationship with another quantization parameter, the Hurst exponent, denoted by $H$ :

$$
D=2-H \text {. }
$$

The Hurst exponent, a parameter that varies between 0 and 1 , is an indicator of the self-similarity and the persistence of the signal [20]. Depending on the value of $H$, the "color" of the movement or signal can be distinguished. So, values of $H=0$ (o equivalently $D=2)$ are associated with white noise, $H=1(D=1)$ indicates a smooth signal, and $H=0.5(D=1.5)$ is related to a Brownian motion or red noise. This means that the higher the value of the fractal dimension (the lower value of $H$ ), the more complex and less predictable the signal. From the point of view of the theory, the exponent is related to a fractional Brownian motion.

To obtain the Hurst exponent (and therefore the fractal dimension), no standard algorithm has been used. Using the definition of Brownian motion, our own algorithm has been developed, and it has been programmed into the Mathematica symbolic manipulation. After a process of adimensionalization of the signal, the algorithm computes the variances of the incremental variables corresponding to different steps. The magnitudes computed are log-log correlated with respect to the time increment. The slope obtained doubles the Hurst exponent.

For the calculation of the parameter $H$, the EEG signal has been denoted as $x(i)$, and different steps $h_{k}$ have been considered to define incremental variables

$$
\psi_{i}^{h_{k}}=x\left(i+h_{k}\right)-x(i)
$$

where $h_{k}=k \delta$ and $\delta$ denotes the sampling interval.

The mean and variance are calculated $v^{h_{k}}$ for each $k$-th incremental variable. If the index supports a fractional Brownian function model, the increments must follow a Gaussian distribution:

$$
\psi_{i}^{h_{k}} \approx \mathcal{N}\left(0, v^{h_{k}}\right)
$$

with variance $v^{h_{k}}$ proportional to $h_{k}^{2 H}, H$ being the Hurst exponent.

Representing the log-log plot of the variances against the lags, data close to a line with slope $2 H$ are obtained. Once $H$ has been calculated, the fractal dimension is obtained directly using the previous relationship $D=2-H$ (See Table 1 and Figure 1 ).

Table 1. Example of the correlation coefficients of the regression for the computation of the Hurst parameter in the six electrodes (F3, F4, O1, O2, T3, T4).

\begin{tabular}{cc}
\hline Channel & Correlation Coef. \\
\hline F3 & 0.988259 \\
\hline F4 & 0.990549 \\
\hline O1 & 0.990549 \\
\hline O2 & 0.987347 \\
\hline T3 & 0.989813 \\
\hline T4 & 0.980922 \\
\hline
\end{tabular}




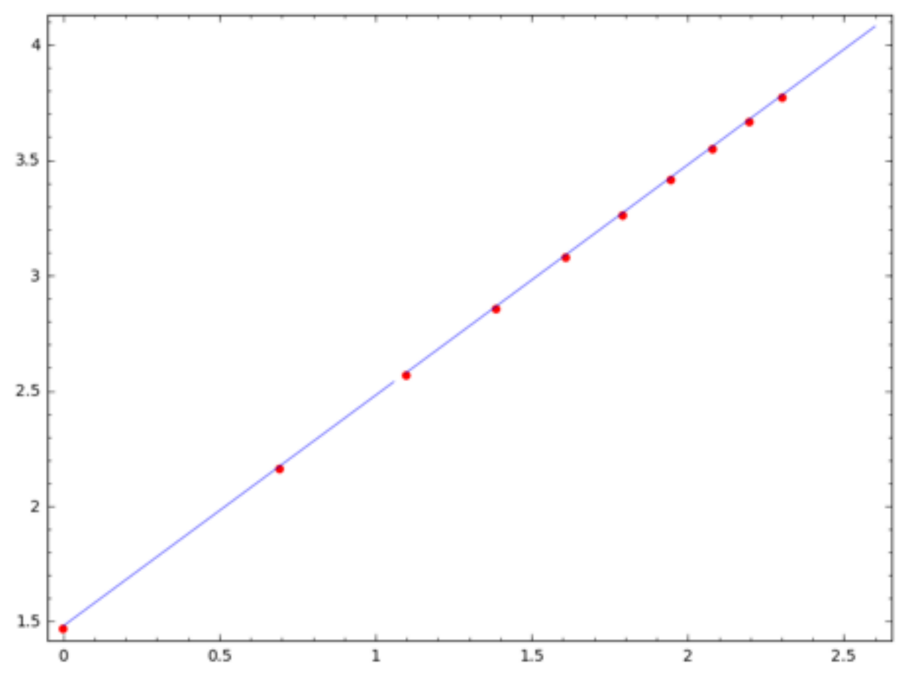

Figure 1. Log-log plot of the variances as function of the steps $\left(h_{k}, v^{h_{k}}\right)$ at an EEG signal in the simulation of driving a military vehicle task in Afghanistan scenery.

\subsection{Data Collection and Analysis}

The initial sample for the experiment was composed of 41 volunteers from the Armed Forces, NCOs, and Professional Military of Troops and Marines (MPTM). The subjects were recruited from the instructors of the "San Gregorio" National Training Center (CENAD) and the students being trained at this center to obtain special driving licenses. The sample included right-handed subjects with no neurological history and who were not taking chronic medication, with no history of brain damage, vision problems, or learning difficulties.

Due to failures in the data collection/simulator system, 9 participants were discarded, which resulted in a final sample of 32 subjects ( $30.6 \pm 6.5$ years; range $22-45,2$ female). Of these, 10 of them were driving instructors, and 22 were students who wanted to obtain the special driving license for the "LMV Lince" vehicle (a Spanish light multipurpose vehicle).

The electroencephalographic tests were collected at the "San Gregorio" National Training Center (CENAD), in states of rest (with eyes closed and open) and during the use of the dynamic simulation platform for the LMV Lince vehicle driver instruction. Several driving scenarios, of different difficulty, were recreated including settings in Mali (desert scenario, flat, monotonous, and of low complexity), Afghanistan (highly complex scenario in mountainous areas with embankments), and a track training circuit with different driving exercises (medium complexity). In the first two scenarios, the subjects drove freely, while on the training track, they received constant external instructions on the exercises and maneuvers they had to perform. The SOMNOwatch plus EEG-6 portable electroencephalograph (Somnomedics, Germany) was used to collect EEG signals. Channels F3, F4, T3, T4, O1, and O2 were recorded according to the Jasper International 10-20 System, referenced to Cz. Filters with cutoff frequencies 0.5 and $70 \mathrm{~Hz}$ were applied. The sampling frequency was $256 \mathrm{~Hz}$. The EEG recording was segmented into five epochs of 3 min each: closed eyes (ce), open eyes (oe), Mali (M), Afghanistan (Af), and track (P). From each epoch, a $30 \mathrm{~s}$ segment was analyzed. Extensive high- and low-frequency pass filters were applied to avoid artifacts.

Once the segments to be analyzed had been selected, the data were processed using codes designed by our team in the Mathematica symbolic manipulator, reconstructing the EEG signal and calculating the fractal dimension as indicated in Section 2.

Once the fractal dimension values were obtained for each subject, electrode, and EEG type, the data were statistically analyzed. An exploratory data analysis was performed, and a parametric $\mathrm{T}$ test was applied for related samples to contrast the hypothesis that the difference in means between the different types of EEG was zero in any of the 6 electrodes with a confidence level of $95 \%(\alpha=0.05)$ and of $99 \%(\alpha=0.01)$. The Kolmogorov- 
Smirnov tests were previously used to verify the data normality hypothesis and Levene's for homoscedasticity or equality of variances.

To visualize the obtained results, multitasking maps were made using Duchon splines.

\section{Results}

After analyzing the data, we could establish that the fractal dimension takes values in a range between 1.14 and 1.69. Table 2 shows the average values of the dimension in each channel for each type of EEG as well as the cortical average in the six channels.

Table 2. Average values of the fractal dimension in each channel (F3, F4, O1, O2, T3, and T4) for each type of EEG, basal states with closed eyes (ce) and open eyes before and after the simulations (oe and oe2), and military vehicle driving simulation tasks (AF, M, P), $(n=32)$. The last line shows the cortical average of the six channels for each type of EEG.

\begin{tabular}{ccccccc}
\hline \multicolumn{7}{c}{ FRACTAL DIMENSION } \\
\hline CHANNEL & ce & oe & Af & M & P & OE2 \\
\hline F3 & $1.435 \pm 0.092$ & $1.435 \pm 0.079$ & $1.401 \pm 0.069$ & $1.420 \pm 0.073$ & $1.404 \pm 0.058$ & $1.412 \pm 0.080$ \\
F4 & $1.374 \pm 0.070$ & $1.389 \pm 0.060$ & $1.351 \pm 0.066$ & $1.368 \pm 0.075$ & $1.362 \pm 0.063$ & $1.383 \pm 0.081$ \\
O1 & $1.236 \pm 0.059$ & $1.317 \pm 0.051$ & $1.403 \pm 0.063$ & $1.370 \pm 0.064$ & $1.388 \pm 0.060$ & $1.315 \pm 0.049$ \\
O2 & $1.243 \pm 0.067$ & $1.324 \pm 0.057$ & $1.400 \pm 0.071$ & $1.369 \pm 0.069$ & $1.387 \pm 0.069$ & $1.322 \pm 0.053$ \\
T3 & $1.321 \pm 0.085$ & $1.391 \pm 0.084$ & $1.404 \pm 0.089$ & $1.373 \pm 0.082$ & $1.406 \pm 0.069$ & $1.382 \pm 0.084$ \\
T4 & $1.344 \pm 0.079$ & $1.398 \pm 0.086$ & $1.437 \pm 0.100$ & $1.414 \pm 0.088$ & $1.448 \pm 0.083$ & $1.391 \pm 0.074$ \\
Cortical Mean & $1.326 \pm 0.076$ & $1.376 \pm 0.071$ & $1.399 \pm 0.078$ & $1.386 \pm 0.076$ & $1.399 \pm 0.068$ & $1.368 \pm 0.071$ \\
\hline
\end{tabular}

The graphic representation of the computed values helps to understand them. Thus, Figure 2 shows the average values of the dimension in each of the six electrodes (F3, F4, O1, O2, T3, T4) for each of the EEG types (baseline with closed eyes (ce), baseline with open eyes (oe), Afghanistan-type driving (Af), Mali-type driving (M), and training track (P)). It was observed how the dimension has a similar behavior in the frontal zone for all types of EEG. In the occipital zone, the values of the fractal quantifier were lower for the basal states, increasing during driving simulation tasks. In the temporal area, there appeared to be a difference between the EEG in a state of rest with closed eyes (where the lowest values occur) and the rest of the types of EEG.

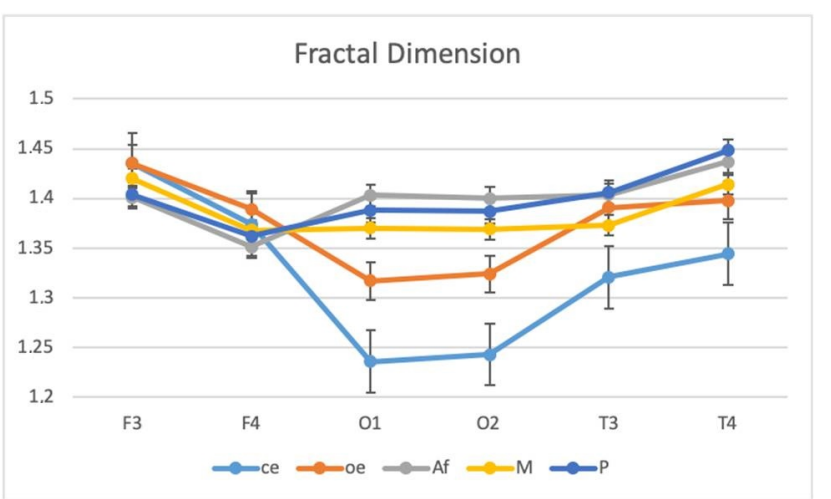

Figure 2. Average values of the fractal dimension in each electrode (F3, F4, O1, O2, T3, and T4) for the five types of EEG (closed eyes (ce), open (oe), Afghanistan-type driving (Af), Mali-type driving $(\mathrm{M})$, and training track $(\mathrm{P})$ ).

The analysis of the evolution of the fractal dimension when passing from basal EEG types to conductions with different levels of difficulty has been separated by areas. In the occipital area, $\mathrm{O} 1$ and $\mathrm{O} 2$ electrodes, it is appreciated how the quantifier increased as the test or type of EEG became more complex. The fractal dimension increased due to the opening of the eyes (change from the basal state with closed eyes to baseline with open eyes) and especially when starting the driving simulations and leaving the resting state. An 
increase in the parameter was observed when driving became more complex (going from the Mali scenario to the Afghanistan one) together with a slight decrease when returning to the training track where the level of difficulty was medium. The right temporal zone (T4) showed a clear increase in dimension with the evolution of the task. In the left zone (T3), a decrease was observed when passing from the baseline state with open eyes to the driving test in Mali (see Figure 3).

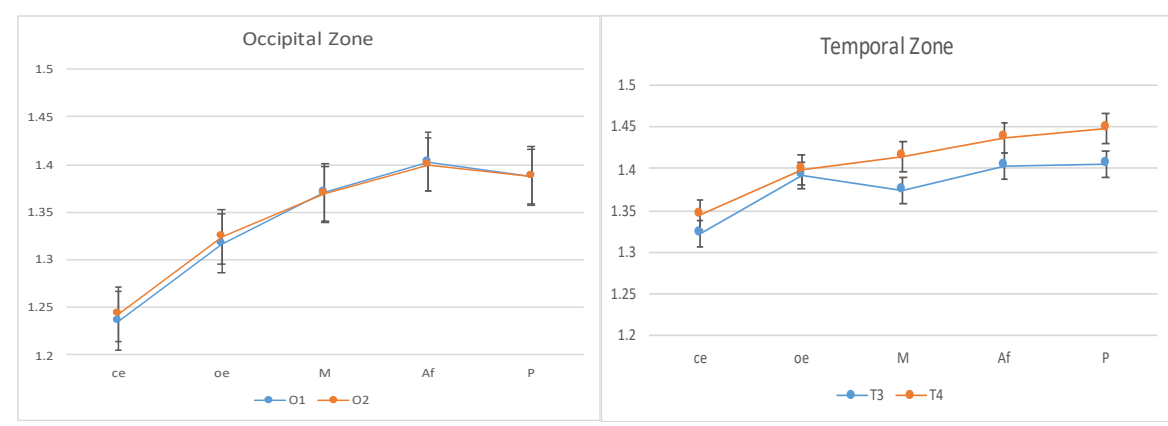

Figure 3. Fractal dimension values in the different types of EEG (closed eyes (ce), open (oe), Afghanistan type conduction (Af), Mali type conduction (M), and training track (P)) in the occipital area (left) and temporal zone (right).

Figure 4 shows the evolution in the frontal area. In this case, the average of the dimension remained more stable than in the rest of the areas, decreasing slightly with the complexity of the simulation tasks. The frontal area behaves in an inverse way to the previous ones.

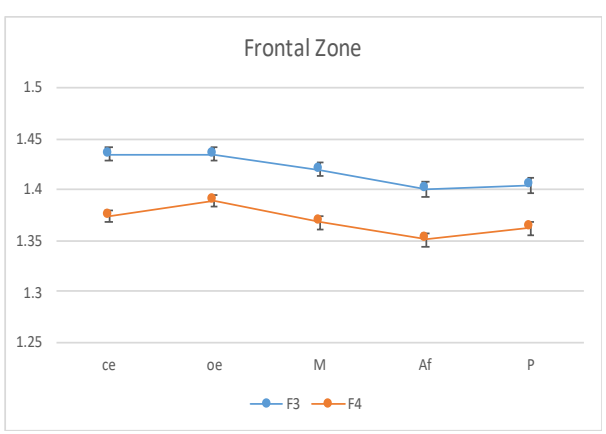

Figure 4. Fractal dimension values in the different types of EEG (closed eyes (ce), open (oe), Afghanistan-type driving (Af), Mali-type driving (M), and training track (P)) in the frontal area $(\mathrm{F} 3, \mathrm{~F} 4)$.

The previous results can be seen in the multitasking map in Figure 5, where the five types of EEG are represented on the horizontal axis and the six electrodes are represented on the vertical axis. The highest values of the fractal dimension were observed in the frontal zone in the basal states and in the temporal and occipital zone during the driving simulation tests.

Once the exploratory analysis was carried out, the hypothesis test was applied to compare the pairs of EEG types. The significant differences found are shown in Table 3. The entire temporal and occipital zones showed significant differences in the comparisons between open and closed eyes and also when comparing the basal state of closed eyes with all the driving simulation tasks. 


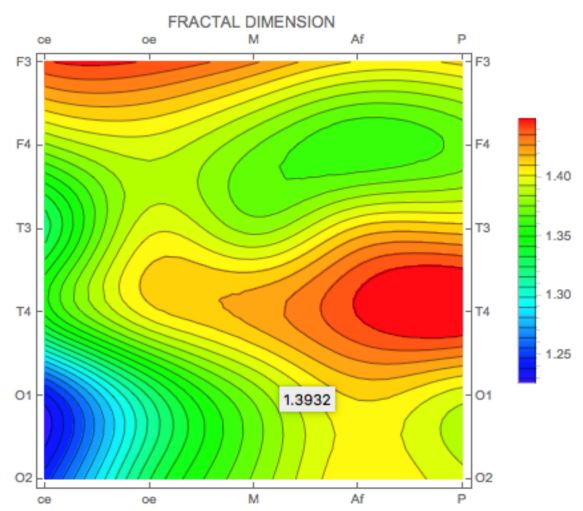

Figure 5. Multitask map of the fractal dimension in the five types of EEG ( $X$-axis) and in the six analyzed electrodes ( $Y$-axis).

Table 3. $p$-values of the pairwise comparison hypothesis test of the EEG types in each channel (F3, $\mathrm{F} 4, \mathrm{O} 1, \mathrm{O} 2, \mathrm{~T} 3$, and T4) for the fractal dimension. The values of $p<0.001$ are highlighted in red color, $0.01<p<0.001$ in yellow color, and $0.01<p<0.05$ in green color. (The meaning of the abbreviatures is given in page 4 ).

\begin{tabular}{ccccccccccc}
\hline & \multicolumn{10}{c}{ FRACTAL DIMENSION $(\boldsymbol{p}$-Values $)$} \\
\hline & oe-ce & A-ce & M-ce & P-ce & A-oe & M-oe & P-oe & A-M & P-M & P-A \\
\hline F3 & 0.983 & 0.091 & 0.473 & 0.099 & 0.060 & 0.001 & 0.053 & 0.139 & 0.201 & 0.551 \\
\hline F4 & 0.122 & 0.164 & 0.734 & 0.407 & 0.009 & 0.178 & 0.040 & 0.216 & 0.656 & 0.174 \\
\hline O1 & 0.000 & 0.000 & 0.000 & 0.000 & 0.000 & 0.000 & 0.000 & 0.002 & 0.062 & 0.023 \\
\hline O2 & 0.000 & 0.000 & 0.000 & 0.000 & 0.000 & 0.000 & 0.000 & 0.004 & 0.079 & 0.022 \\
\hline T3 & 0.000 & 0.000 & 0.004 & 0.000 & 0.370 & 0.160 & 0.221 & 0.028 & 0.006 & 0.885 \\
\hline T4 & 0.000 & 0.000 & 0.000 & 0.000 & 0.033 & 0.226 & 0.002 & 0.083 & 0.006 & 0.352 \\
\hline
\end{tabular}

If these simulations are compared with the basal state with eyes open, it is observed that the occipital area continued to present differences in all cases and in the rest of the areas they varied for each task.

Comparing the types of pairs EEG during the driving simulations, significant differences were obtained between driving in the Mali and Afghanistan scenarios in the occipital zone and in part of the temporal (O1, O2, and T3) one. Comparing driving on the track and driving in Mali, differences were obtained in the temporal zone (T3, T4) and when comparing the track with the Afghanistan scenario, the occipital zone presented the most significant differences. Figure 6 shows graphically the significant differences found in these comparisons.

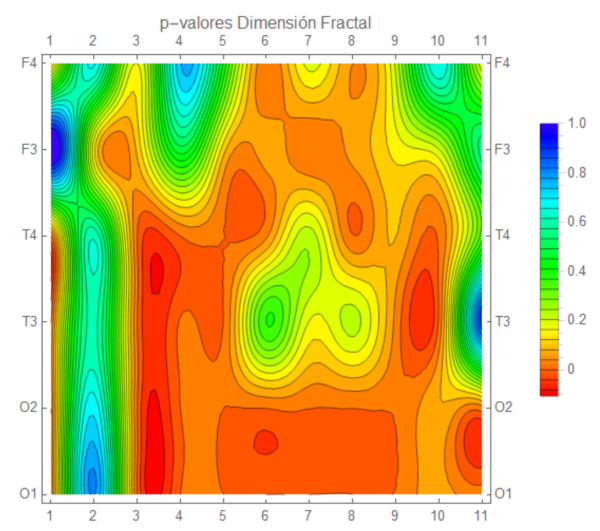

Figure 6. $p$-values obtained in the pairwise comparison hypothesis test of the EEG types (X-axis) in each channel ( $Y$-axis). 


\section{Discussion}

The study of brain activity during the performance of tasks that require attention and vigilance is usually carried out using the classical techniques of EEG frequency band analysis. In general, brain activities show increased alpha, beta, and gamma power in the frontal and central areas in attention tasks with increased theta, alpha, and beta activity in vigilance tasks. During visual attention, beta activity improves, and the low-frequency phase synchronization of the EEG is commonly shown to increase between the frontal and parietal regions of the brain in tasks that require attentional orientation [21].

Several authors have reported the existence of cortical and subcortical brain networks involved in the variation of the EEG power spectra while performing flight tasks [22,23]. This classical analysis can be extended by applying nonlinear dynamics quantifiers, fractal techniques, and new spectral methods. For example, Yang et al. [24] used the correlation dimension to quantify changes in brain activity when performing seven types of EEG.

In previous works, our group has explored the brain activity during the simulation of military vehicle driving using quantifying parameters calculated by our own methods. [25]. In this case, the conduction simulations were performed on an iPad, and the EEG recordings were recorded with a hospital EEG. These indices have been shown to be useful for quantifying brain activity, varying in the areas involved in the performance of the different tasks.

The use of driving simulators (with a high degree of analogy to real situations) allows the attentional process developed to carry out the cognitive function to mobilize the responses associated with the task and to activate the brain areas where the neurocognitive process occurs. This activation has been registered with the increase of the fractal dimension. Specifically, this parameter has revealed the activation of the primary visual area located in the occipital lobe and of the primary auditory cortex located in the temporal lobe, when moving from a basal state to driving simulation tasks, confirming the hypothesis raised. The decrease in dimension in the frontal area during the performance of the tasks can be explained by the appearance of slow activity in this area because the task requires the activation of the rest of the brain areas, which are responsible for sensory processing (auditory and visual).

The increase in the dimension in the posterior brain areas when passing from rest with closed eyes to rest with open eyes can be explained by the blockage of the alpha rhythm produced under normal conditions when the eyes are opened, which suggests adequate cortical reactivity.

The exhaustive filtering carried out on the signal before its analysis has made the maximum average values in the activity zones lower than those obtained in previous works, where the applied filters were not so restrictive.

The results presented in this work indicate that the fractal dimension is a good indicator of brain activity, which can detect the brain bioelectric changes produced in the areas involved in carrying out the different tasks of military vehicle driving simulation, increasing with its complexity, which is a fact that confirms the hypothesis.

With this study, our team validates the results obtained in previous works with quantifiers such as the normalized slope descriptors as well as with the analysis of the mean frequency, where the differences related to gender were analyzed. Thus, we can conclude that when faced with the same task, the female group presented higher values of brain activation than the male group [26].

Our results confirm the paradigm of chaos theory, which states that apparently erratic phenomena (in this case, brain waves) present a hidden geometry, which is well modeled by fractal theory. The fractal dimension proves to be an effective quantifying parameter for natural records (bioelectrical, climatic, etc.) and also for evolutionary social and economic phenomena.

Author Contributions: Conceptualization, M.A.N. and M.V.S.; methodology, M.A.N. and M.V.S.; software, M.A.N., M.V.S. and A.O.; validation, C.D.-P., L.L.D.S. and M.Á.I.; formal analysis, C.R.; 
investigation, all team; resources, M.V.S.; data curation, M.V.S. and C.R.; writing-original draft preparation, M.V.S.; writing-review and editing, M.A.N. and M.V.S.; visualization, M.V.S.; supervision, M.A.N. and M.V.S.; project administration, M.V.S.; funding acquisition, M.V.S. All authors have read and agreed to the published version of the manuscript.

Funding: This reseach was funded by Centro Universitario de la Defensa de Zaragoza, projects 2019-04, 2020-13.

Institutional Review Board Statement: The study complies with the provisions of the Biomedical Research Law (Law 14/2007, of July 3, on biomedical research) and is governed by the principles agreed in the Declaration of Helsinki. The research protocol and informed consent were approved by the Clinical Research Ethics Committee of the General Defense Health Inspection (Resolution act 09/18, code 58/18). Each participating subject signed their corresponding patient information and informed consent sheets before performing the test. The subjects also completed the army health questionnaire (Annex to Instruction 22/2008 of January 31).

Informed Consent Statement: Informed consent was obtained from all subjects involved in the study.

Data Availability Statement: See the Institutional Review Board Statement.

Acknowledgments: Projects CUD ID: 2019-04 and 2020-13.

Conflicts of Interest: The authors declare no conflict of interest.

\section{References}

1. Micheloyannis, S.; Vourkas, M.; Bizas, M.; Simos, P.; Stam, C.J. Changes in linear and nonlinear EEG measures as a function of task complexity. Brain Topogr. 2003, 15, 239-247. [CrossRef] [PubMed]

2. Namazi, H.; Kulish, V.V. Fractional diffusion based modelling and prediction of human brain response to external stimuli. Comput. Math. Methods Med. 2015, 2015, 148534. [CrossRef] [PubMed]

3. Blanco, S.; García, H.; Quiroga, R.Q.; Romanelli, L.; Rosso, O.A. Stationarity of the EEG series. IEEE Eng. Med. Biol. 1995, 14, 395-399. [CrossRef]

4. He, B.; Astolfi, L.; Valdés-Sosa, P.A.; Marinazzo, D.; Palva, S.O.; Bénar, C.G.; Michel, C.M.; Koenig, T. Electrophysiological brain connectivity: Theory and implementation. IEEE Trans. Biomed. Eng. 2019. [CrossRef] [PubMed]

5. Rioul, O.; Vetterli, M. Wavelets and Signal Processing. IEEE Signal Process. Mag. 2001, 8, 14-38. [CrossRef]

6. Theiler, J.; Rapp, P. Re-examination of the evidence for low-dimensional, nonlinear structure in the human electroencephalogram. Electroenceph. Clin. Neurophysiol. 1996, 98, 213-222. [CrossRef]

7. Mandelbrot, B.B.; Pignoni, R. The Fractal Geometry of Nature; WH Freeman: Ney York, NY, USA, 1983 ; Volume 173.

8. Paramanathan, P.; Uthayakumar, R. Application of fractal theory in analysis of human electroencephalographic signals. Comput. Biol. Med. 2008, 38, 372-378. [CrossRef] [PubMed]

9. Phothisonothai, M.; Nakagama, M. EEG signal classification method based on fractal features and neural network. In Proceedings of the Engineering in Medicine and Biology Society, EMBS 2008, 30th Annual International Conference of the IEEE, Vancouver, BC, Canada, 20-24 August 2008; pp. 3880-3883.

10. Namazi, H.; Daneshi, A.; Azarnoush, H.; Jafari, S.; Towhidkhah, F. Fractal based analysis of the influence of auditory stimuli on eye movements. Fractals 2018, 26, 1850040. [CrossRef]

11. Zappasodi, F.; Olejarczyk, E.; Marzetti, L.; Assenza, G.; Pizzella, V.; Tecchio, F. Fractal dimension of EEG activity senses neuronal impairment in acute stroke. PLoS ONE 2014, 9, e100199. [CrossRef]

12. Finotello, F.; Scarpa, F.; Zanon, M. EEG signal features extraction based on fractal dimension. In Proceedings of the 37th Annual International Conference of the IEEE Engineering in Medicine and Biology Society (EMBC), Milano, Italy, $25-29$ August 2015.

13. Ruiz-Padial, E.; Ibáñez-Molina, A.J. Fractal dimension of EEG signals and heart dynamics in discrete emotional states. Biol. Psychol. 2018, 137, 42-48. [CrossRef] [PubMed]

14. Navascués, M.A.; Sebastián, M.V. Fitting curves by fractal interpolation: An application to the quantification of cognitive brain processes. In Thinking in Patterns: Fractals and Related Phenomena in Nature; Novak, M.M., Ed.; World Scientific Publishers: Singapore, 2004; pp. 143-154.

15. Sebastián, M.V.; Navascués, M.A. A relation between fractal dimension and Fourier transform. Electroencephalographic study using spectral and fractal parameters. Int. J. Comput. Math. 2008, 85, 657-665. [CrossRef]

16. Navascués, M.A.; Sebastián, M.V.; Valdizán, J.R. Fractal and smooth complexities in electroencephalographic processing. J. Appl. Comput. Math. 2015, 4, 1-6.

17. Campbell, K.; Bell, I.; Bastien, C. Evoked potential measures of information processing during natural sleep. In Sleep, Arousal and Performance; Broughton, R.J., Ogilvie, R.D., Eds.; Birkhäuser: Boston, MA, USA, 1992; pp. 88-116.

18. Navascués, M.A.; Chand, A.K.B.; Viswanathan, P.; Sebastián, M.V. Fractal interpolation functions: A short survey. Spec. Issue Fractal Theory Appl. Appl. Math. 2014, 5, 1834-1841. [CrossRef] 
19. Navascués, M.A.; Sebastián, M.V. Construction of affine fractal functions close to classical interpolants. J. Comp. Anal. Appl. 2007, 9, 271-285.

20. Hurst, H.E.; Black, R.P.; Simaika, Y.M. Long-Term Storage: An Experimental Study; Constable: London, UK, 1965.

21. Dombrowe, I.; Hilgetag, C.C. Occipitoparietal alpha-band responses to the graded allocation of top-down spatial attention. J. Neurophysiol. 2014, 112, 1307-1316. [CrossRef] [PubMed]

22. Dussault, C.; Jouanin, J.C.; Philippe, M.; Guezennec, C.Y. EEG and ECG changes during simulator operation reflect mental workload and vigilance. Aviat. Space Environ. Med. 2005, 76, 344-351. [PubMed]

23. Borghini, G.; Vecchiato, G.; Toppi, J.; Astolfi, L.; Maglione, A.; Isabella, R.; Caltagirone, C.; Kong, W.; Wei, D.; Zhou, Z.; et al. Assessment of mental fatigue during car driving by using high resolution EEG activity and neurophysiologic indices. In Proceedings of the 2012 Annual International Conference of the IEEE Engineering in Medicine and Biology Society, San Diego, CA, USA, 28 August-1 September 2012; pp. 6442-6445.

24. Yang, H.; Wang, Y.; Wang, C.J.; Tai, H.M. Correlation dimensions of EEG changes during mental tasks. In Proceedings of the 26th Annual International Conference of the IEEE EMBS, San Francisco, CA, USA, 1-5 September 2004; pp. 616-619.

25. Idiázabal-Alecha, M.A.; Sebastián-Guerrero, M.V.; Navascués-Sanagustín, M.A.; Arcos-Sánchez, C.; Arana-Aritméndiz, M.V.; Ruiz-López, C.; Iso-Pérez, J.M. A cortical study of the attention in military simulation test. Rev. Neurol. 2018, 66, 331-339. [PubMed]

26. Sebastián, M.V.; Arana, M.V.; Sánchez, C.; Navascués, M.A.; Idiázabal, M.A.; Ruiz, C.; Iso, J.M. Attention processes in military simulation tasks: Gender-associated differences. Rev. Cient. Soc. Esp. Enferm. Neurol. 2018, 48, 2-8. [CrossRef] 